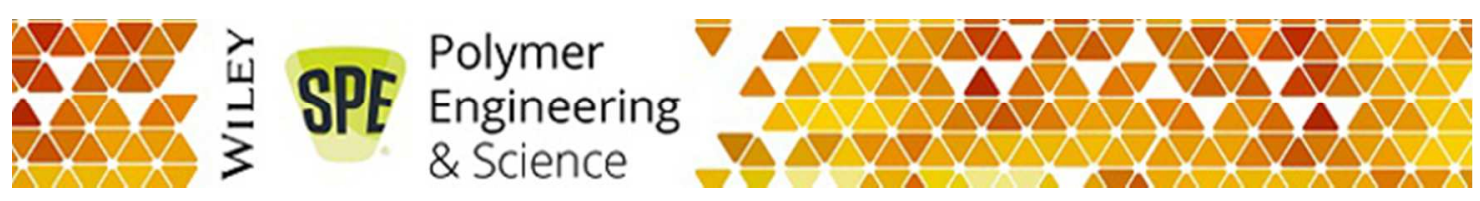

\title{
Fracture toughness of acrylic resins: viscoelastic effects and deformation mechanisms
}

\begin{tabular}{|r|l|}
\hline Journal: & Polymer Engineering \& Science \\
\hline Manuscript ID & PES-17-0132.R1 \\
\hline Wiley - Manuscript type: & Research Article \\
\hline Date Submitted by the Author: & 27-Feb-2017 \\
\hline Complete List of Authors: & $\begin{array}{l}\text { Pini, Tommaso; Politecnico di Milano, Chemistry, Materials and Chemical } \\
\text { Engineering } \\
\text { Briatico-Vangosa, Francesco; Politecnico di Milano, Dipartimento di } \\
\text { Chimica, Materiali e Ingegneria Chimica "G. Natta" } \\
\text { Frassine, Roberto; Politecnico di Milano, Chemistry, Materials and Chemical } \\
\text { Engineering } \\
\text { Rink, Marta; Politecnico di Milano, Dipartimento di Chimica Industriale e } \\
\text { Ingegneria Chimica Giulio Natta }\end{array}$ \\
\hline Keywords: & \begin{tabular}{l} 
fracture, viscoelastic properties, thermoplastics, toughness, damage zone \\
\hline
\end{tabular} \\
\hline
\end{tabular}




\title{
TITLE
}

Fracture toughness of acrylic resins: viscoelastic effects and deformation mechanisms

\author{
AUTHORS \\ Tommaso Pini ${ }^{1}$, Francesco Briatico-Vangosa ${ }^{1}$, Roberto Frassine ${ }^{1}$, Marta Rink ${ }^{1}$ \\ ${ }^{1}$ Politecnico di Milano, Department of Chemistry, Materials and Chemical Engineering, Piazza \\ Leonardo da Vinci 32, 20133, Milano, Italy \\ tommaso.pini@polimi.it
}




\section{ABSTRACT}

The time dependence of fracture toughness of two different acrylic resins, one plain and one toughened, intended to be used as continuous fiber composite matrices was studied. By performing fracture tests following the fracture mechanics approach, the energy release rate, $\mathrm{G}_{\mathrm{Ic}}$, was determined at different temperatures and displacement rates and by applying the time-temperature superposition it was possible to obtain $\mathrm{G}_{\mathrm{Ic}}$ as a function of crack speed, $\dot{a}$, over a wide range of speeds. The trends obtained for the two resins were different. For the plain resin it could be well described by J. G. Williams' viscoelastic fracture theory while for the toughened resin, the trend obtained was attributed to a change in the damage mechanism occurring at the crack tip during fracture. From measurements of the process zone size it was deduced that the damage mechanism at the crack tip for the plain resin was the same irrespective of time and temperature, for the toughened resin instead, different mechanisms seem to take place. This hypothesis was supported by results of volume strain measurements in tensile tests at different temperature and strain rates. 


\section{INTRODUCTION}

Acrylic glassy polymers have been widely used in many different applications. These polymers are stiff, transparent and have very good weathering properties but generally yield by crazing and show brittle fracture. In order to increase the fracture toughness of polymeric materials, not only acrylics, a common practice is to add rubber particles into the material. The increase in fracture toughness is then related to size, type and concentration of the rubber particles [1-3].

It is possible to prepare acrylic polymers by in-situ polymerization and this has promoted their use in many applications such as bone cement in orthopedics and dentures, cavity filling and other in dentistry. A more recent development of such thermoplastic polymers is their use as matrices for continuous fiber composite materials since: (i) in comparison to thermoset resins they are more ductile and tough, show better weldability, recyclability and have infinite shelf-life; (ii) some of the processing technologies relevant to thermosetting resins such as infusion and resin transfer molding can be also applied to acrylics overcoming one of the main drawback of other thermoplastic polymers i.e. their high viscosity even at high temperatures and thus the difficulty in fiber impregnation and processing technologies.

In structural applications of polymeric materials their viscoelastic nature must be taken into consideration in order to guarantee a reliable performance under different loading conditions. In this paper fracture toughness, which is one of the main properties to be assessed for material selection and product design, will be considered. Several theories have been proposed and reviewed in the literature which apply the fracture mechanics approach to viscoelastic materials [4-7]. Such theories, taking into account the time dependence of the mechanical properties of polymers, generally describe both, the fracture initiation stage by relating fracture toughness with crack initiation time and the fracture propagation stage relating toughness with crack propagation speed. Concerning toughened polymers, the time dependence of mechanical properties may result altered with respect to that of the relevant homopolymers [8-10]. 
In the present work two thermoplastic acrylic resins, one plain and one toughened with rubber, were studied. The fracture toughness dependence on crack propagation speed was determined and results were compared with viscoelastic fracture mechanics theories predictions. The toughened resin was more deeply investigated to clarify the role of the damage mechanisms occurring at crack tip on the time dependence of fracture toughness. Fracture behavior of continuous fiber reinforced composites based on the same resins was also studied considering the effects of the viscoelasticity of the relevant matrices and the results obtained will be reported in a separate paper.

\section{MATERIALS}

Two different acrylic resins developed by Arkema were studied, namely:

- $\quad$ Elium ${ }^{\circledR}$, named E, having a glass transition temperature, $T_{g}$, of $127^{\circ} \mathrm{C}$.

- Elium Impact ${ }^{\circledR}$, named EI, which is toughened with about $10 \mathrm{wt} \%$ of an acrylic block copolymer (Nanostrength $\left.{ }^{\circledR}\right)$, which produces nanostructures having a size below $50 \mathrm{~nm}$. EI shows two glass transitions at $-25^{\circ} \mathrm{C}$ and $130{ }^{\circ} \mathrm{C}$ respectively.

Specimens for mechanical tests were prepared by casting monomers into a closed mold. Elium samples were let polymerize for 24 hours at room temperature and then a thermal treatment of 1 hour at $80{ }^{\circ} \mathrm{C}$ and 1 hour at $120^{\circ} \mathrm{C}$ was applied to complete polymerization. Elium Impact samples polymerization was performed with a thermal cycle of 5 hours at $80{ }^{\circ} \mathrm{C}$ and 1.5 hours at $125^{\circ} \mathrm{C}$.

\section{TEST METHODS}

\section{Dynamic Mechanical Analysis}

Tests were performed on a TA RSA-3 machine in three-point bending test configuration adopting prismatic specimens having dimensions of $26 \mathrm{~mm} \times 4 \mathrm{~mm} \times 1 \mathrm{~mm}$. Isothermal tests at temperatures 
between -60 and $110{ }^{\circ} \mathrm{C}$ and at frequencies from 0.1 to $10 \mathrm{~Hz}$ were carried out on both resins. The storage modulus master curves and the relevant shift factors were obtained by shifting the isothermal curves horizontally along the logarithmic time axis.

\section{Tensile tests}

Uniaxial tensile tests were performed on both resins adopting dumbbell specimens having a gage of $18 \mathrm{~mm} \times 5 \mathrm{~mm} \times 2 \mathrm{~mm}$; tests were carried out at constant displacement rates of $0.1,1$ and 10 $\mathrm{mm} / \mathrm{min}$ and temperatures of $0,23,40$ and $60^{\circ} \mathrm{C}$. One specimen was tested for each condition. Stress was evaluated as the ratio between the load and the initial cross-section. Strains were measured with Digital Image Correlation (DIC) adopting Vic-2D software on tests video recordings. In order to apply this method, the specimens were first painted white and then a fine black pattern was sprayed before testing. Water based paints were chosen to avoid any kind of interaction with the material.

Yield stress as a function of time to yield was determined; the yield point was taken at the maximum in the stress-strain curves. In the case of E resin, at low temperature and high rate, some specimens broke without reaching a maximum and in such cases the highest stress reached was considered as yield stress, as a first approximation.

During the tests, the volume change was also determined to investigate deformation mechanisms that may take place in the materials. Under the assumption of transversal isotropy, the lateral contraction is the same in the two transversal directions, therefore it is possible to evaluate the volume strain, $\Delta V / V_{0}$, as

$$
\frac{\Delta V}{V_{0}}=(1+\varepsilon)\left(1+\varepsilon_{\text {lat }}\right)^{2}-1
$$

in which $\varepsilon$ and $\varepsilon_{\text {lat }}$ are the longitudinal and lateral strains respectively. Tests were performed on an Instron 1121 dynamometer equipped with a $10 \mathrm{kN}$ load cell and a thermostatic cabinet. 


\section{Fracture tests}

Fracture toughness, evaluated as the strain energy release rate, $G_{I c}$, was determined as a function of crack speed, $\dot{a}$, adopting the double torsion test configuration (Fig. 1 (a)). This test allows to control crack propagation speed and is therefore very suitable to study fracture toughness rate dependence [11-13]. In this work specimens having dimensions of $120 \mathrm{~mm}$ x $45 \mathrm{~mm}$ x $6 \mathrm{~mm}$ (initial notch length, a, $22.5 \mathrm{~mm}$ ) and $200 \mathrm{~mm} \times 70 \mathrm{~mm}$ x $10 \mathrm{~mm}$ (initial notch length $30 \mathrm{~mm}$ ) were adopted. In both cases side grooves having a depth which was $15 \%$ of the thickness, $b$, were introduced to prevent crack deviation from center line.

Strain energy release rate, $G_{I c}$, is given by

$$
G_{I C}=\frac{P_{c}^{2}}{2 b_{c}} \frac{\partial C}{\partial a}
$$

in which $P_{c}$ is the load during crack propagation, $b_{c}$ is the thickness of the grooved cross-section, $C$ is the specimen compliance and $a$ the crack length. In this configuration, specimen compliance is expected to be linear with crack length and in a test at constant displacement rate, $\dot{x}$, crack propagation takes place at a constant load. Under these conditions, crack propagation speed, $\dot{a}$, can be evaluated as

$$
\dot{a}=\frac{\dot{x}}{P_{c} \frac{\partial C}{\partial a}}
$$

Since $\partial C / \partial a, \dot{x}$ and $P_{c}$ are constant, the crack propagation speed, $\dot{a}$, is constant as well. To obtain $\partial C / \partial a$ compliance calibration was performed by sequentially introducing different notch lengths on a single specimen and measuring the relevant compliance at $23{ }^{\circ} \mathrm{C}$ and $1 \mathrm{~mm} / \mathrm{min}$. The value of $\partial C / \partial a$ for the different testing conditions was properly scaled taking into account the ratio between the compliance of the specimen of the fracture test and that of the calibration specimen. 
Fracture tests were carried out at temperatures from 0 to $60{ }^{\circ} \mathrm{C}$ and displacement rates varying from 0.1 to $100 \mathrm{~mm} / \mathrm{min}$. From 1 to 5 specimens were adopted for each testing condition. Corrections proposed by Leevers [14] to take into account large displacements were applied. Double torsion is a very convenient technique to study crack propagation while it is not suitable for crack initiation analysis. Therefore, fracture tests were also carried out adopting the three-point bending test configuration (Fig. 1 (b)). With this configuration crack initiation phase may be analyzed and, further, optical observation and Digital Image Correlation analysis at the crack tip could be performed. Specimens having dimensions of $90 \mathrm{~mm}$ x $20 \mathrm{~mm}$ x $10 \mathrm{~mm}$ (initial notch length, a, 10 $\mathrm{mm}$ ) were tested at a constant displacement rate of $5 \mathrm{~mm} / \mathrm{min}$ and at temperatures varying from 0 to $60{ }^{\circ} \mathrm{C} . G_{I C}$ at crack initiation, was evaluated following [15]:

$$
G_{I c}=\frac{U_{c}}{b W \varphi(a / W)}
$$

where $U_{c}$ is the input energy up to crack initiation, $b$ and $W$ are the thickness and width respectively and $\varphi$ is a calibration factor, reported in [15], dependent on crack length, $a$. $G_{I c}$ was evaluated at crack initiation, but tests were video recorded during crack propagation as well and this stage was analyzed with Digital Image Correlation too. Fracture tests were performed on an Instron 1185 dynamometer equipped with a $10 \mathrm{kN}$ load cell and a thermostatic cabinet and on a MTS 831.50 servohydraulic dynamometer at Montanuniversität in Leoben, Austria.

\section{RESULTS AND DISCUSSION}

\section{Viscoelastic effects on fracture}

Fig. 2 (a) shows the typical load displacement curve for a double torsion test before and after the correction for large displacement: after this correction a practically constant load is obtained throughout the test. Fig. 2 (b) shows fracture toughness, $G_{I c}$, as a function of crack propagation speed, $\dot{a}$, at four different temperatures for E resin. Applying the time-temperature equivalence, it 
was possible to extend the crack propagation speed range: the isothermal curves were shifted along the logarithmic time axis, so as to obtain a good superposition of the data, as has been previously done in [16-18]. Fig. 3 (a) and Fig. 3 (b) show the master curves so obtained at the reference temperature of $23{ }^{\circ} \mathrm{C}$ for $\mathrm{E}$ and $\mathrm{EI}$ resins respectively. For $\mathrm{E}$ resin $G_{I C}$ increases as crack speed increases as expected from viscoelastic fracture theories while, for the toughened resin EI, an opposite trend is observed. Results for E resin were fitted with a power law in order to discuss them in the framework of Williams' viscoelastic fracture theory $[6,7]$.

Following this theory, based on the assumption of a constant crack tip opening displacement and on a power law dependence for both relaxation modulus as a function of time and yield stress as a function of time to yield, fracture toughness can be expressed as

$$
G_{I c}=\delta_{c} \sigma_{y}\left(t_{\alpha}\right)
$$

in which $\delta_{c}$ is the crack tip opening displacement and $\sigma_{y}$ the yield stress evaluated at a certain characteristic time $t_{\alpha}$ which is the time necessary for a crack to propagate, at constant speed $\dot{a}$, across the process zone length, $\alpha$, ahead of the crack tip:

$$
t_{\alpha}=\frac{\alpha}{\dot{a}}
$$

The yield stress vs. time to yield can be expressed as a power law having an exponent $-m$; equation (5) then becomes

$$
G_{I c}=\delta_{c} \sigma_{0} t_{\alpha}^{-m}
$$

Adopting Dugdale's model [19] the process zone length $\alpha$ can be written as

$$
\alpha=\frac{\pi}{8} \frac{G_{I c} E}{\sigma_{y}^{2}}
$$


Expressing also the modulus $E$ as a power law with an exponent $-n$, and combining equations (6), (7) and (8) it is possible to obtain the relationship between strain energy release rate and crack propagation speed:

$$
G_{I C} \propto \dot{a}^{n^{\prime}}=\dot{a} \frac{m}{1+n-m}
$$

In the power law so obtained the exponent $n^{\prime}$ is related to $n$ and $m$, the exponents of the power laws describing relaxation modulus as a function of time and yield stress as a function of time to yield respectively.

The relaxation modulus was obtained from DMA tests. Fig. 4 (a) shows isothermal curves of storage modulus, $E^{\prime}$, as a function of frequency, $v$, for $E$ resin. By shifting the isothermal curves along the logarithmic frequency axis, the master curve for E resin of $E$, shown in Fig. 4 (b), was obtained. The master curve for EI resin, obtained with the same procedure, is also shown in Fig. 4 (b). The relaxation modulus, $E(t)$, was then approximated as the storage modulus, considering $t=1 /\left(\pi^{2} v\right)[20]$. Relaxation modulus vs. time curves for both polymers are reported in Fig. 5 (a). The exponent $n$ was evaluated as the slope of the linear fit of the data in a double logarithmic plot, over a time scale from $10^{-4}$ to $10^{6} \mathrm{~s}$. This time scale covers the crack propagation characteristic times, $t_{\alpha}$, relevant to double torsion tests. Such times were evaluated from the process zone lengths (see next section) and the crack propagation speeds occurring under the different test conditions.

As for the exponent $m$ in eq. (6), it was obtained from tensile tests results. Fig. 6 (a) reports yield stress vs. time to yield at four different temperatures for E resin. The master curve, shown in Fig. 6 (b), was obtained by shifting isothermal curves along the logarithmic time axis so as to obtain the best superposition of the data. The master curve relevant to EI resin, obtained with the same procedure, is also reported in Fig. 6 (b). The exponent $m$ was evaluated as the slope of the linear fit of the data in a double logarithmic plot. The crack propagation characteristic times, $t_{\alpha}$, relevant to some test conditions were outside the fitting range from Fig. 6 (b), and the value of yield stress was in such cases extrapolated. 
Tangent modulus and the relevant times at a fixed strain of $1 \%$ were also determined. Data were shifted applying the shift factors obtained building the yield stress master curves and are reported, as plus (+) symbols, on the relaxation modulus curves in Fig. 5 (a). A good agreement was found, indicating the adequacy of the approximation made to obtain the relaxation modulus.

Table 1 reports the values of the exponents $n, m$ and $n^{\prime}$ (eq. (9)) along with the experimental value of $n^{\prime}$ obtained from Fig. 3 (a). In the case of $E$ resin there is a fair agreement between the value predicted by Williams' theory and the experimental one. As for the toughened material EI obviously no comparison was considered since a decreasing trend was found.

It is worth to notice that the shift factors obtained building the master curves of conservative part of complex moduli, yield stress and fracture toughness, reported in Fig. 5 (b) in an Arrhenius type plot for the two resins, are very similar to each other as previously found in $[16,17]$.

\section{Process zone size}

The size of the process zone was measured on single edge notched specimens in three-point bending tests. Crack tip opening displacement was measured by DIC. Referring to the schematic in Fig. 7 (a), the following procedure was adopted: (i) the $v$ displacement in the $y$ direction was measured at a certain distance $x_{1}$ from the crack tip and an example of the dependence of $v$ on the distance from the crack plane in the $y$ direction found is shown in Fig. 7 (b). (ii) The two branches of the $v$ displacement vs. $y$ were linearly fitted and the distance between the intercepts was taken as the displacement jump at the crack plane and named $\delta$. (iii) was measured at several distances from the crack tip obtaining a curve as in the example of Fig. 7 (c). The crack tip opening displacement (CTOD or $\delta_{c}$ ) is the value of $\delta$ at the crack tip (i.e. $x=0$ ). The distance between the crack tip and the coordinate $x$ at which $\delta$ becomes constant can be taken as the length of the process zone, $\alpha$. This procedure was carried out at crack initiation and at several stages of crack propagation, moving 
the reference system to the position of the crack tip as in Fig. 7 (a). Results are reported in Fig. 8 (a) for both resins. It can be observed that for both resins $\delta_{c}$ is constant throughout the test. As regards temperature, for the untoughened resin $\mathrm{E}, \delta_{c}$ is constant and values are in agreement with those present in literature for similar materials $[21,22]$ for which fracture occurs by the propagation of a single craze ahead of the crack tip.

For resin EI instead, $\delta_{c}$ increases as temperature increases. The average values of $\delta_{c}$ are reported in Fig. 8 (b) as a function of temperature. Similar results were obtained for the process zone length, $\alpha$, and Fig. 8 (c) reports the average values of $\alpha$ as a function of temperature for both resins. In the case of $\mathrm{E}$ resin, the fact that the crack tip opening displacement is almost constant over the range of temperature investigated is in agreement with the assumptions of Williams' viscoelastic fracture theory, while this does not occur in the case of EI resin. A verification of the results obtained from DIC, was made by comparing the values of the strain energy release rate at fracture initiation obtained from eq. (4) and from eq. (10) which is equivalent to eq. (5) for crack initiation:

$$
G_{I c}=\delta_{c} \sigma_{y}\left(t_{\text {init }}\right)
$$

in which $\delta_{c}$ is the crack tip opening displacement and $\sigma_{y}\left(t_{\text {init }}\right)$ is the yield stress at the time of crack initiation $t_{\text {init }}$, which was evaluated from the power laws fitted to data in Fig. 6 (b). It can be observed from Fig. 9 that a fair agreement was found.

The variation of $\delta_{c}$ and $\alpha$ with temperature for EI resin was thought to be due to a change in the damage mechanisms occurring at the crack tip during the fracture process.

\section{Damage mechanisms in the toughened EI resin}

In the case of plain $\mathrm{E}$ resin, since the size of the process zone does not change neither with temperature nor with displacement rate it is reasonable to suppose that the damage mechanism is 
always the same. As mentioned above, this mechanism is probably a single craze ahead of the crack tip.

In a toughened polymer different damage mechanisms can occur: multiple crazing, in which a high number of crazes develop as a consequence of the stress state around the dispersed phase and cavitation of the rubber particles which promotes the shear yielding of the surrounding matrix [2325]. Cavitation followed by shear yielding are accompanied by higher levels of energy dissipation in comparison to multiple crazing $[23,26]$. An attempt to observe the damage mechanisms via SEM, as previously done (see for example $[27,28]$ ), was not successful in the present work. Nevertheless, since these different mechanisms are accompanied by different changes in volume strain, indications on the deformation mechanisms occurring in polymeric materials was derived from the analysis of the volumetric strain during tensile tests as previously reported in the literature [8, 26,29-31]. The slopes of volumetric strain vs. longitudinal strain plots give an indication of which mechanism is acting. In the ideal case of pure crazing the slope should be equal to 1 while in the case of pure shear yielding equal to 0 . Cavitation is also accompanied by volume changes but much smaller than in the case of crazing. Different mechanisms can be due to different material properties, or, for the same material, to different testing conditions such as temperature and strain rate. Fig. 10 shows volumetric strain vs. longitudinal strain curves for EI resin obtained from tensile tests performed at various temperatures and strain rates. It is possible to observe that all curves have a similar initial slope related to elastic volume strain and then after a certain point, which was observed to be practically coincident with the maximum of the stress-strain curves, there is an increase in slope. Different slopes, always between 0 and 1 , were found. This indicates that there could be more than one of the aforementioned damage mechanisms acting at the same time. Nevertheless, as strain rate increases or temperature decreases slopes tend to 1 indicating that probably multiple crazing prevails with respect to cavitation and shear yielding. These results may give an explanation to the trend of fracture toughness master curve found for EI resin, even if it should be pointed that the stress state at the crack tip is not the same as in a tensile test. The higher 
toughness observed at lower crack propagation speeds could be associated with cavitation and shear yielding observed at low strain rates during tensile tests, while the lower values of toughness at higher crack propagation speeds could be associated to multiple crazing which was found to become the main mechanism at higher strain rates in tensile tests.

\section{CONCLUSIONS}

The fracture behavior of two thermoplastic acrylic resins, one plain and one rubber toughened, was investigated. Fracture toughness as a function of crack propagation speed was measured over a wide range of crack speeds thanks to the applicability of the time-temperature superposition. The two materials show different trends, fracture toughness increases as crack speed increases for the untoughened resin E while the opposite is observed for the toughened resin EI. DIC performed around the crack tip during fracture tests showed that:

(i) in the case of the untoughened resin (E) the process zone size at the crack tip does not vary as temperature increases (or crack speed decreases) indicating that the mechanisms occurring at the crack tip do not change, it can therefore be thought that the strain energy release rate dependence on crack speed is governed by the viscoelastic properties of the bulk material as predicted by viscoelastic fracture theories. Indeed, results obtained are in good agreement with Williams' theory;

(ii) for the toughened resin the process zone size increases as temperature increases (or crack speed decreases) indicating that a change in the local deformation mechanisms at the crack tip occurs. This conclusion was supported by the results of volume strain measurements performed in tensile tests carried out at different rates and temperatures which showed that a change of the damage mechanisms takes place at different conditions of temperature and displacement rates. The variation of the energy dissipated locally because of the change in the damage mechanism masks the viscoelastic effects of the bulk material. 


\section{ACKNOWLEDGEMENTS}

Authors would like to thank Arkema Company and the Groupement de Recherche du Lacq, France for the materials and the support provided and Prof. Gerald Pinter, Dr. Steffen Stelzer and the personnel of Montanuniversität of Leoben, Austria, for their help and willingness. 


\section{REFERENCES}

1. C.B. Bucknall, Toughened plastics. Springer Science + Business Media, Dordrecht (1977)

2. R.A. Pearson and A.F. Yee, J. Mater. Sci., 26, 3828 (1991)

3. C.J.G. Plummer, Ph. Béguelin and H.H. Kausch, Polymer, 37, 7 (1996)

4. R.A. Schapery, Int. J. Fract., 11, 141 (1975)

5. R.A. Schapery, Int. J. Fract., 11, 369 (1975)

6. J.G. Williams, “Applications of linear fracture mechanics” in Failure in Polymers, Advances in Polymer Science, 27, (67-120) Springer-Verlag, Berlin Heidelberg (1978)

7. W. Bradley, W.J. Cantwell and H.H. Kausch, Mech. Time-Depend. Mat., 1, 241 (1997)

8. O. Frank and J. Lehmann, Polym. Sci., 264, 473 (1986)

9. Ph. Béguelin, PhD Thesis, EPFL, Lausanne, Switzerland (1996)

10. M. Todo, K. Takahashi, P-Y.B. Jar and Ph. Béguelin, JSME Int J. Ser. A, 42, 585 (1999)

11. A.G., Evans, J. Mater. Sci., 7, 1137 (1972)

12. R. Frassine, T. Riccò, M. Rink, and A. Pavan, J. Mater. Sci., 23, 4027 (1988)

13. A. Shyam and E. Lara-Curzio, J. Mater. Sci., 41, 4093 (2006)

14. P.S. Leevers, J. Mater. Sci. Lett., 5, 191 (1986)

15. ISO 13586:2000, Plastics - Determination of fracture toughness (GIC and KIC) - Linear elastic fracture mechanics (LEFM) approach, International Organization for Standardization (2001)

16. R. Frassine and A. Pavan, Compos. Sci. Technol., 54, 193 (1995)

17. R. Frassine, M. Rink and A. Pavan, Compos. Sci. Technol., 56, 1253 (1996)

18. P. Mariani, R. Frassine, M. Rink and A. Pavan, Polym. Eng. Sci., 36, 2758 (1996)

19. D.S. Dugdale, J. Mech. Phys. Solids, 8, 100 (1960)

20. R.M. Christensen, Theory of Viscoelasticity, Dover, Mineola (2003)

21. H.R. Brown and I.M. Ward, Polymer, 14, 469 (1973)

22. R. Schirrer, J. Mater. Sci., 22, 2289 (1987) 
23. I. Walker and A.A. Collyer, "Rubber toughening mechanisms in polymeric materials" in Rubber toughened engineering plastics, A.A: Collyer, ed., 29-56, Chapman \& Hall, London (1994)

24. C.B. Bucknall and D.R. Paul, Polymer, 50, 5539-5548 (2009)

25. C. Zhou, H. Liu, M. Chen, G. Wu and H. Zhang, Polym. Eng. Sci., 52, 2523 (2012)

26. C.B. Bucknall, I.K. Partridge and M.V. Ward, J. Mater. Sci., 19, 2064 (1984)

27. X.Y. Xu and X.F. Xu, Polym. Eng. Sci., 51, 902 (2011)

28. M. Bashar, U. Sundararaj and P. Mertiny, Polym. Eng. Sci., 54, 1047 (2013)

29. D. Heikens, S.D. Sjoerdsma and W.J. Coumans, J. Mater. Sci., 16, 429 (1981)

30. R. Schirrer, C. Fond and A. Lobbrecht, J. Mater. Sci., 31, 6409 (1996)

31. F. Addiego, A. Dahoun, C. G’Sell, J-M. Hiver and O. Godard, Polym. Eng. Sci., 49, 1198 (2009) 


\section{TABLES}

Table 1 Power laws exponents comparison

\begin{tabular}{|c|c|c|c|c|}
\hline & $n$ & $m$ & $n^{\prime}$ (theory) & $n^{\prime}$ (experimental) \\
\hline E resin & $(4.89 \pm 0.01) 10^{-2}$ & $(7.2 \pm 0.1) 10^{-2}$ & $(7.4 \pm 0.1) 10^{-2}$ & $(6.3 \pm 0.7) 10^{-2}$ \\
\hline EI resin & $(5.10 \pm 0.09) 10^{-2}$ & $(4.7 \pm 0.2) 10^{-2}$ & $(4.7 \pm 0.2) 10^{-2}$ & - \\
\hline
\end{tabular}

\section{FIGURES CAPTIONS}

Fig. 1 (a) Double torsion test configuration. (b) Three-point bending test configuration

Fig. 2 (a) Example of a load-displacement curve of a double torsion test. Dashed line represents raw data, solid line represents data corrected for large displacements. (b) Fracture toughness vs. crack propagation speed isothermal data from double torsion tests for E resin

Fig. 3 Fracture toughness vs. crack propagation speed master curves at the reference temperature of $23{ }^{\circ} \mathrm{C}$ for E resin (a) and EI resin (b). Solid line is a power law fitting, dashed line is just a visual aid

Fig. 4 (a) Storage modulus vs. frequency isothermal curves for E resin. (b) Storage modulus vs. frequency master curves for E (solid symbols) and EI resins (open symbols) at the reference temperature of $23{ }^{\circ} \mathrm{C}$

Fig. 5 (a) Relaxation modulus curves at the reference temperature of $23{ }^{\circ} \mathrm{C}$ for E resin (solid circles) and EI resin (open circles). Solid lines are power laws fittings, plus symbols $(+)$ refer to tensile tests results. (b) Shift factors as a function of temperature obtained at small strains (circles), yield (triangles) and fracture (squares) at the reference temperature of $23{ }^{\circ} \mathrm{C}$ for E resin (solid symbols) and EI resin (open symbols) 
Fig. 6 (a) Yield stress vs. time to yield isothermal curves for E resin. (b) Yield stress vs. time to yield master curve for E (solid symbols) and EI resins (open symbols) at the reference temperature of $23{ }^{\circ} \mathrm{C}$. Solid lines are power laws fittings

Fig. 7 (a) Crack tip reference system at crack initiation and during crack propagation. (b) Measurement method for the displacement jump, $\delta$, in correspondence of the crack plane. (c) Displacement jump at crack plane vs. distance from crack tip, crack tip opening displacement $\delta_{c}$ and length of the process zone $\alpha$

Fig. 8 (a) Crack tip opening displacement measured with DIC at different crack propagation stages for E resin (solid symbols) and EI resin (open symbols). Data refer to temperatures of $0{ }^{\circ} \mathrm{C}$ (stars), $23{ }^{\circ} \mathrm{C}$ (circles), $40{ }^{\circ} \mathrm{C}$ (squares) and $60{ }^{\circ} \mathrm{C}$ (triangles). $\Delta a=0$ represents crack initiation. Solid lines are the average values for each condition. Average values of $\delta_{c}$ (b) and $\alpha$ (c) as a function of temperature for E resin (solid symbols) and EI resin (open symbols)

Fig. 9 Fracture toughness vs. crack initiation time for E resin (solid symbols) and EI resin (open symbols). Circles refer to results from three-point bending tests (eq. (4)), triangles to eq. (10)

Fig. 10 (a) Volumetric strain vs. longitudinal strain curves at different rates and at fixed temperature of $60{ }^{\circ} \mathrm{C}$ for EI resin. (b) Volumetric strain vs. longitudinal strain curves at different temperatures and at fixed rate of $1 \mathrm{~mm} / \mathrm{min}$ for EI resin. Dashed lines represent ideal curves with slopes equal to 1 and 0 


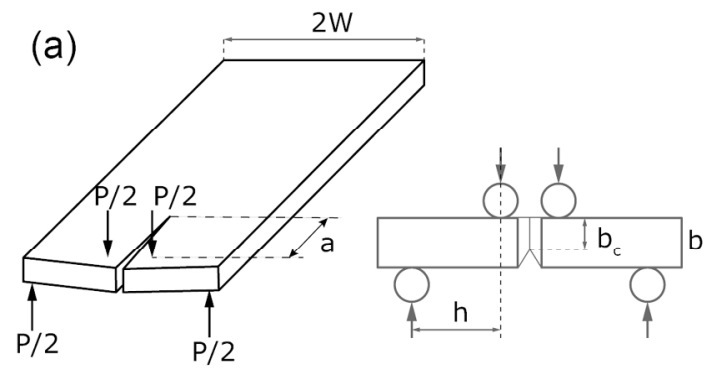

(b)

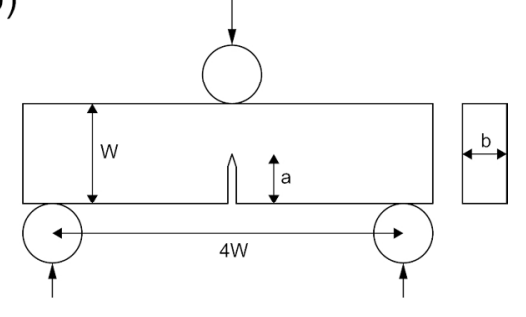

Fig. 1 (a) Double torsion test configuration. (b) Three-point bending test configuration Fig. 1

$170 \times 46 \mathrm{~mm}(300 \times 300 \mathrm{DPI})$ 

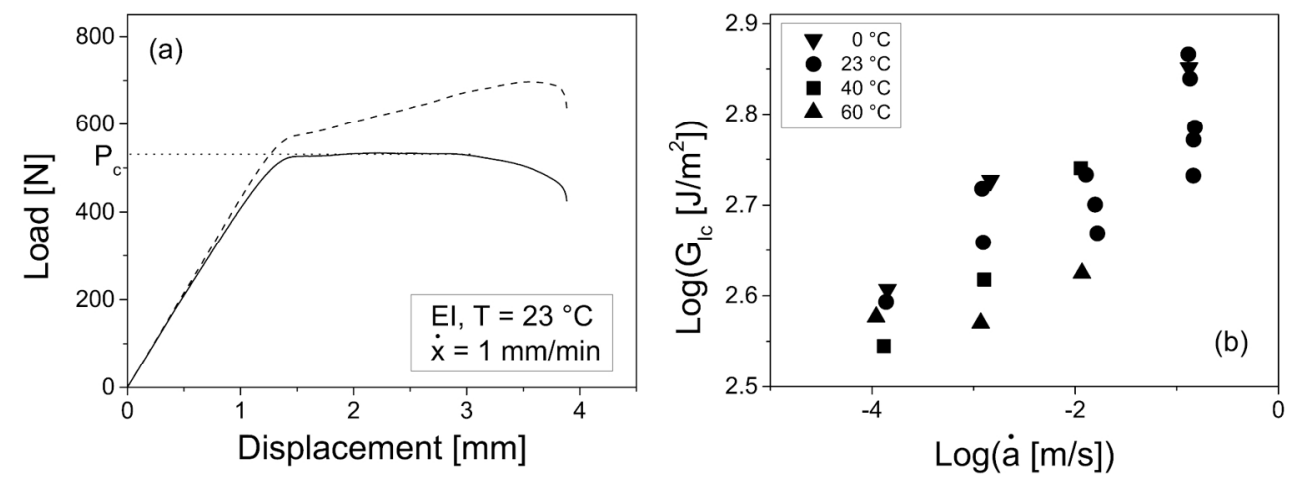

Fig. 2 (a) Example of a load-displacement curve of a double torsion test. Dashed line represents raw data, solid line represents data corrected for large displacements. (b) Fracture toughness vs. crack propagation speed isothermal data from double torsion tests for $E$ resin

Fig. 2

$163 \times 60 \mathrm{~mm}(300 \times 300 \mathrm{DPI})$ 

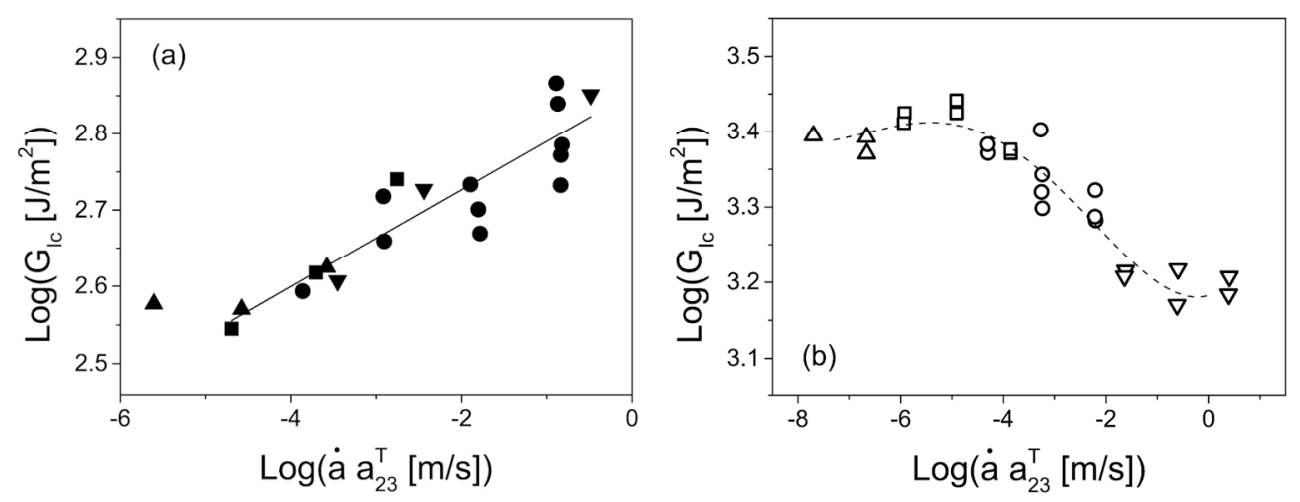

Fig. 3 Fracture toughness vs. crack propagation speed master curves at the reference temperature of $23^{\circ} \mathrm{C}$ for $E$ resin (a) and EI resin (b). Solid line is a power law fitting, dashed line is just a visual aid Fig. 3

$163 \times 62 \mathrm{~mm}(300 \times 300 \mathrm{DPI})$ 

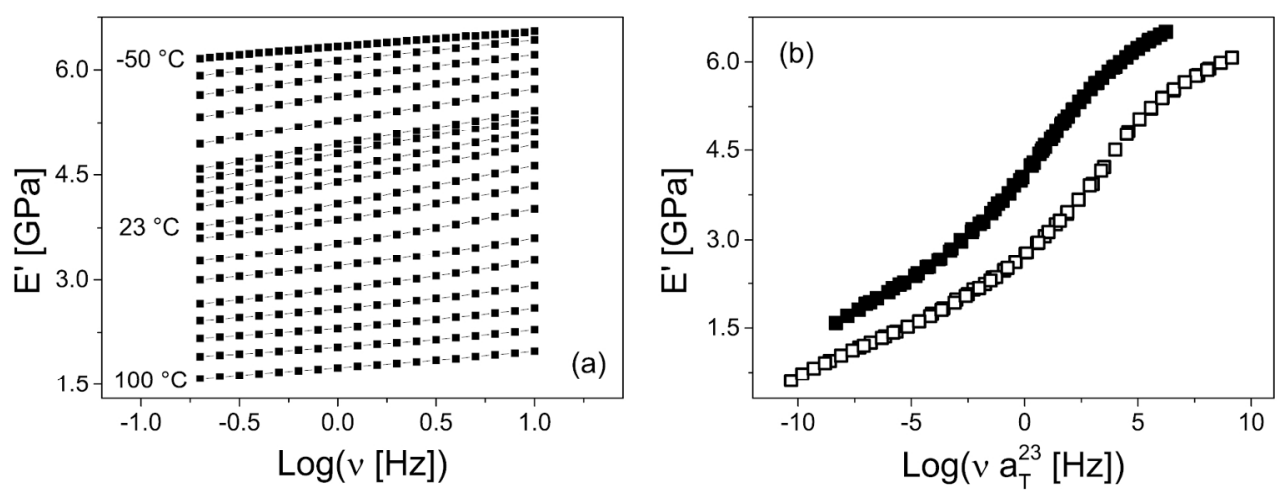

Fig. 4 (a) Storage modulus vs. frequency isothermal curves for E resin. (b) Storage modulus vs. frequency master curves for $\mathrm{E}$ (solid symbols) and EI resins (open symbols) at the reference temperature of $23^{\circ} \mathrm{C}$ Fig. 4

$163 \times 62 \mathrm{~mm}(300 \times 300 \mathrm{DPI})$ 

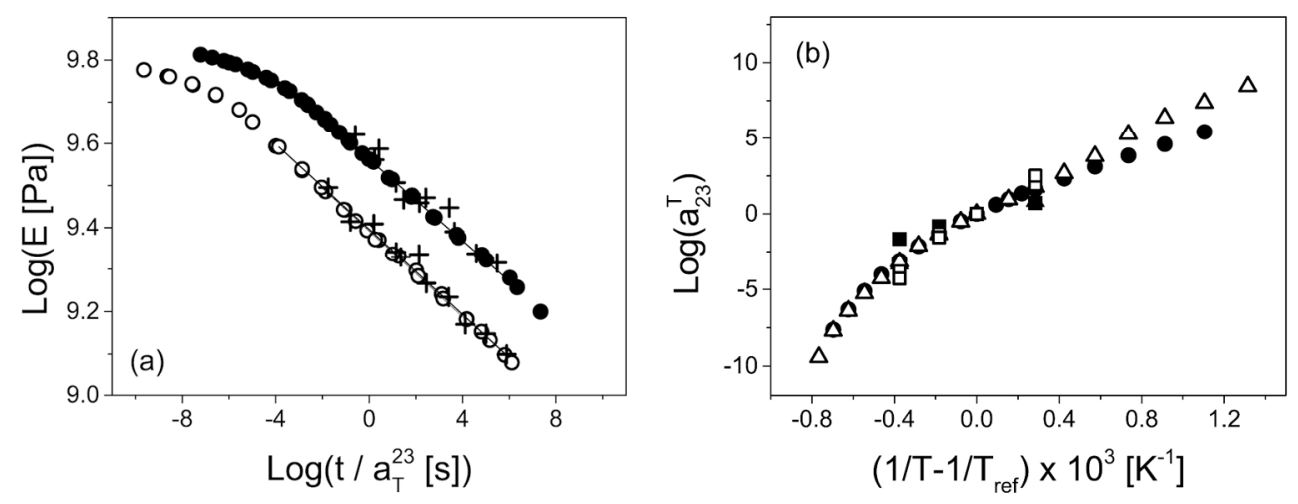

Fig. 5 (a) Relaxation modulus curves at the reference temperature of $23^{\circ} \mathrm{C}$ for $\mathrm{E}$ resin (solid circles) and EI resin (open circles). Solid lines are power laws fittings, plus symbols (+) refer to tensile tests results. (b) Shift factors as a function of temperature obtained at small strains (circles), yield (triangles) and fracture (squares) at the reference temperature of $23^{\circ} \mathrm{C}$ for $\mathrm{E}$ resin (solid symbols) and EI resin (open symbols)

Fig. 5

$163 \times 63 \mathrm{~mm}(300 \times 300 \mathrm{DPI})$ 

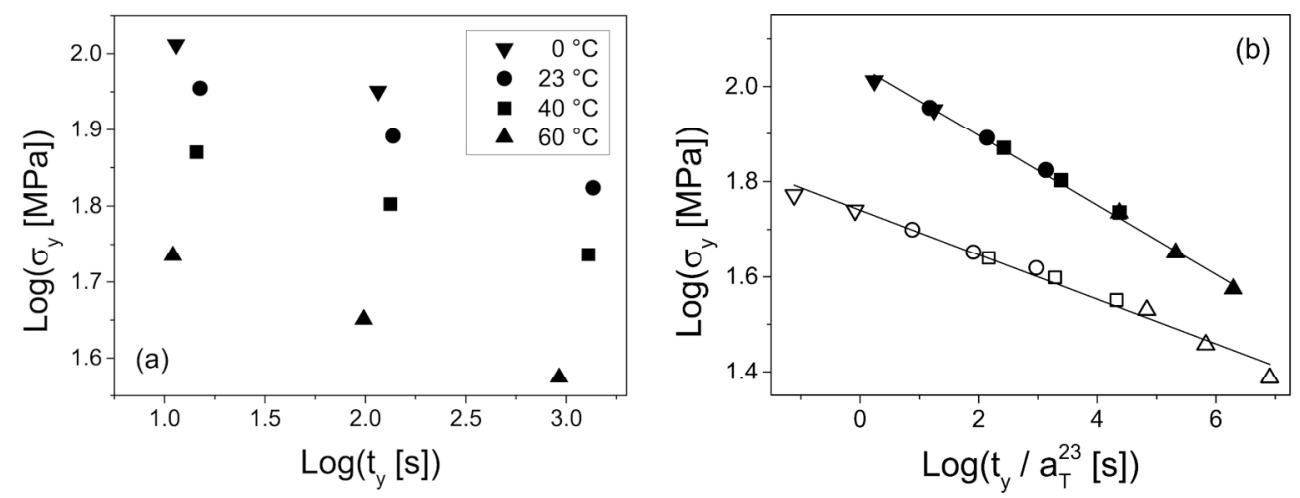

Fig. 6 (a) Yield stress vs. time to yield isothermal curves for E resin. (b) Yield stress vs. time to yield master curve for E (solid symbols) and EI resins (open symbols) at the reference temperature of $23^{\circ} \mathrm{C}$. Solid lines are power laws fittings

Fig. 6

$163 \times 62 \mathrm{~mm}(300 \times 300 \mathrm{DPI})$ 


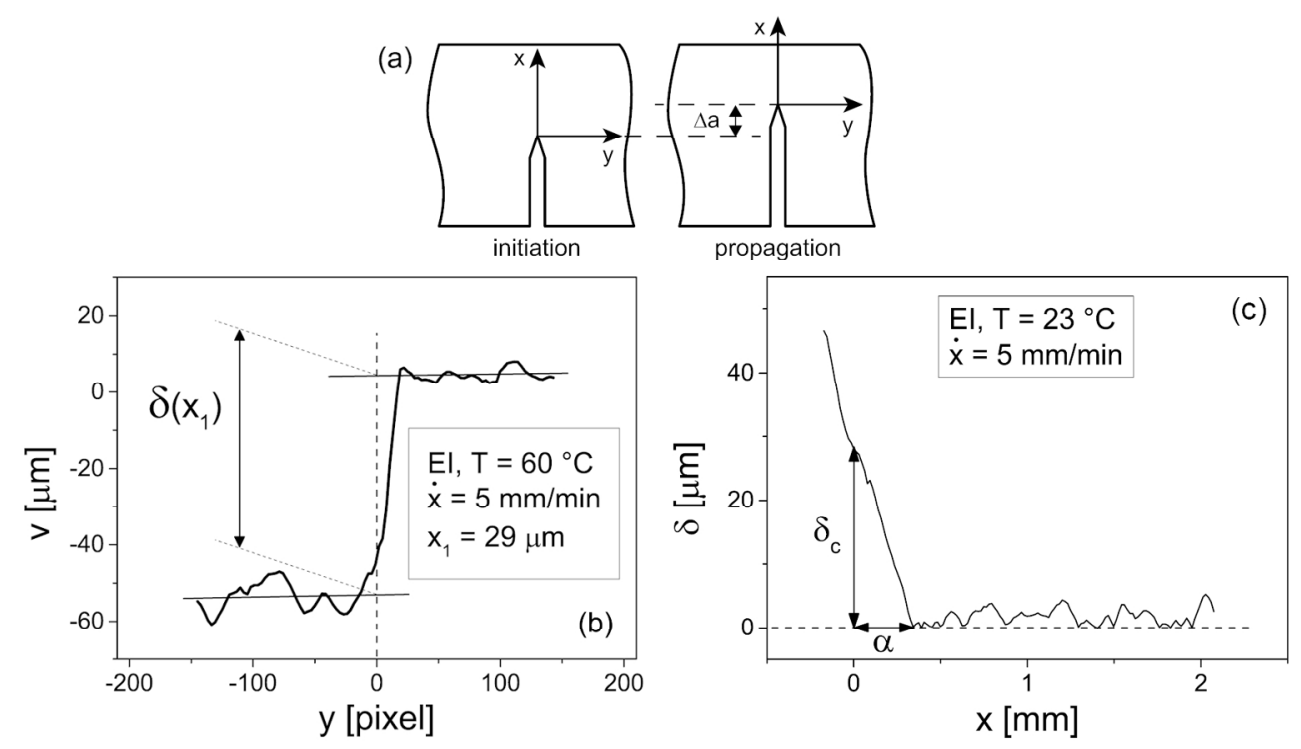

Fig. 7 (a) Crack tip reference system at crack initiation and during crack propagation. (b) Measurement method for the displacement jump, $\delta$, in correspondence of the crack plane. (c) Displacement jump at crack plane vs. distance from crack tip, crack tip opening displacement $\delta_{c}$ and length of the process zone a

Fig. 7

$163 \times 92 \mathrm{~mm}(300 \times 300$ DPI $)$ 

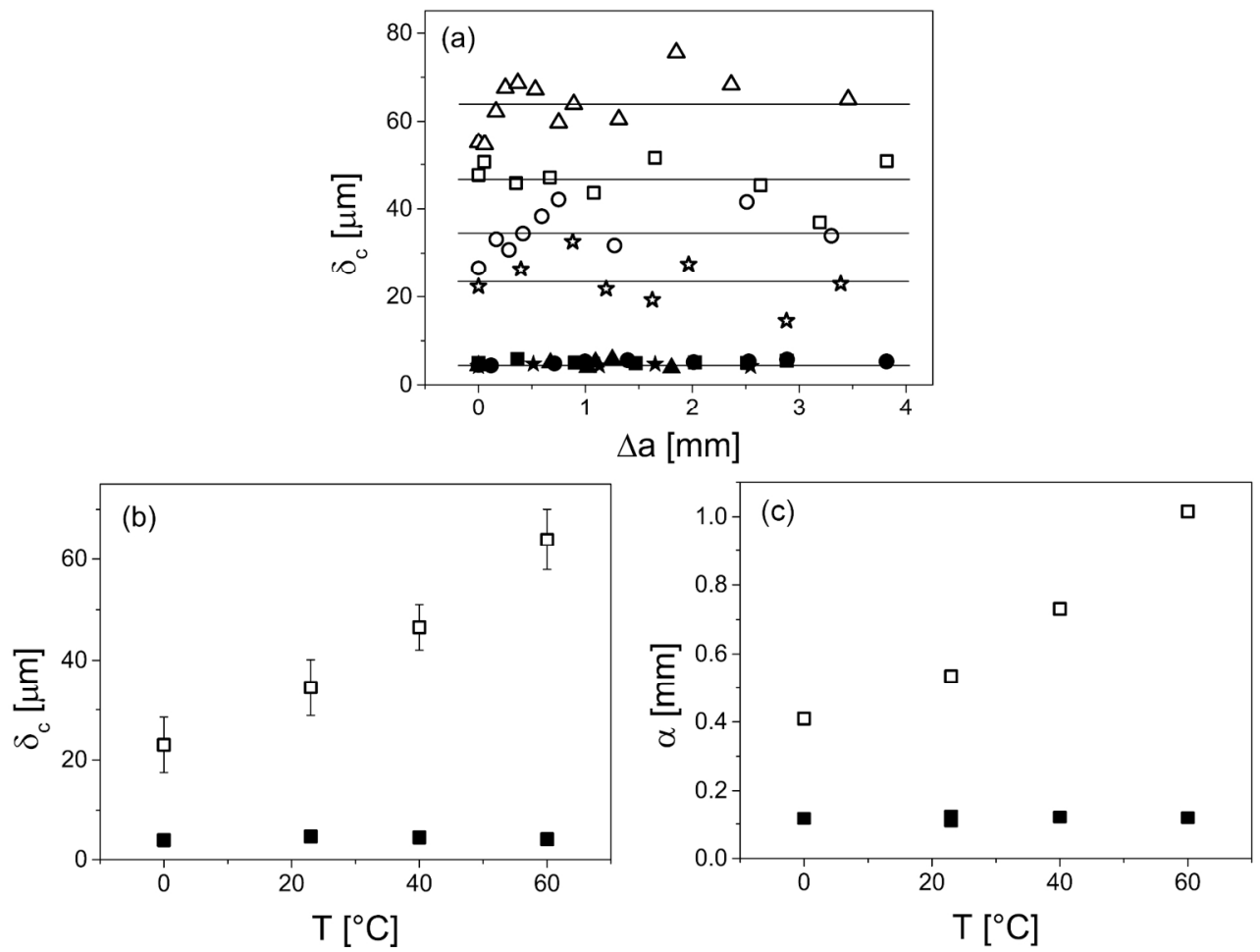

Fig. 8 (a) Crack tip opening displacement measured with DIC at different crack propagation stages for $\mathrm{E}$ resin (solid symbols) and EI resin (open symbols). Data refer to temperatures of $0{ }^{\circ} \mathrm{C}$ (stars), $23{ }^{\circ} \mathrm{C}$ (circles), $40^{\circ} \mathrm{C}$ (squares) and $60^{\circ} \mathrm{C}$ (triangles). $\Delta a=0$ represents crack initiation. Solid lines are the average values for each condition. Average values of $\delta_{c}(b)$ and a (c) as a function of temperature for $E$ resin (solid symbols) and EI resin (open symbols)

Fig. 8

$163 \times 122 \mathrm{~mm}(300 \times 300 \mathrm{DPI})$ 


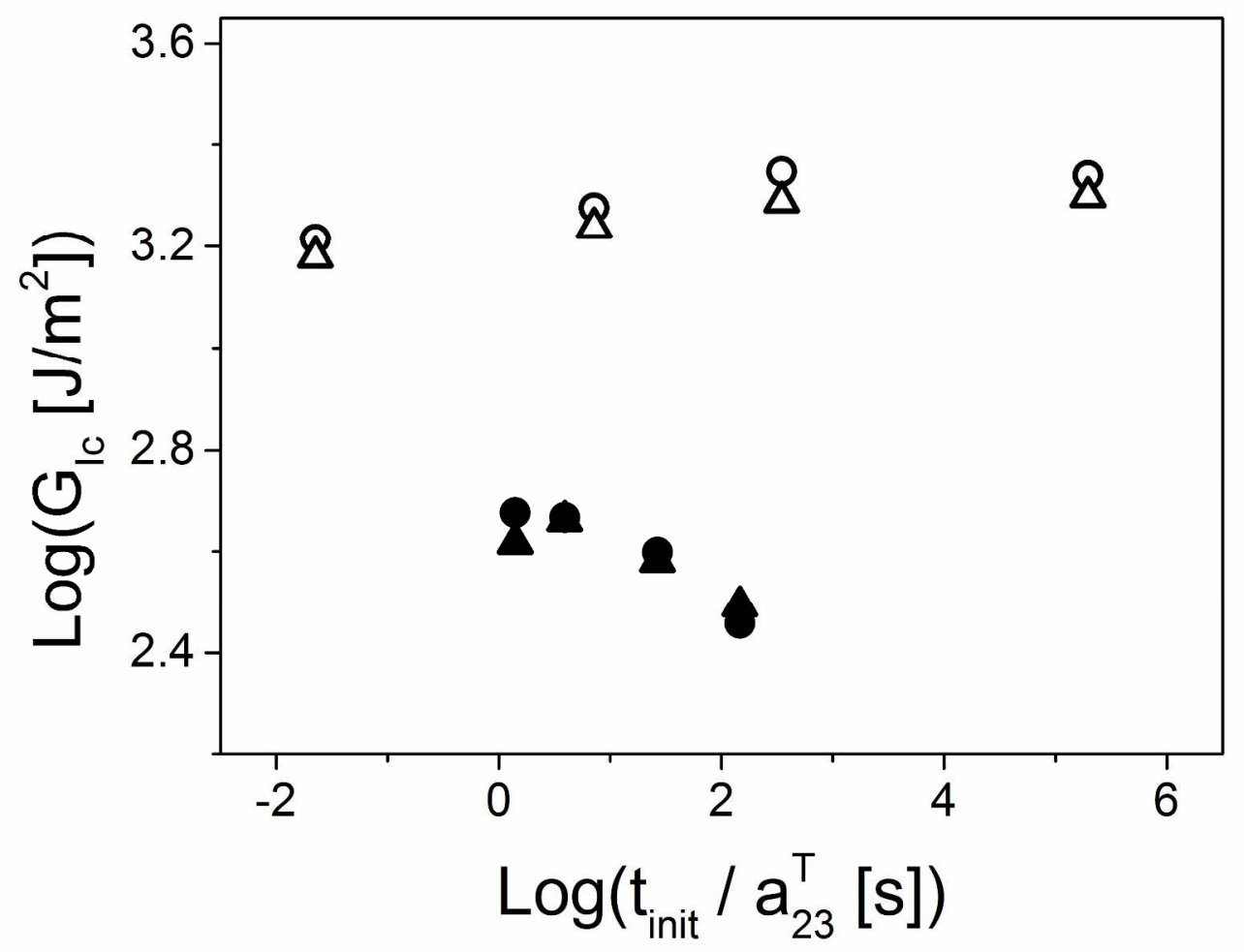

Fig. 9 Fracture toughness vs. crack initiation time for E resin (solid symbols) and EI resin (open symbols). Circles refer to results from three-point bending tests (eq. (4)), triangles to eq. (10)

Fig. 9

$247 \times 191 \mathrm{~mm}(300 \times 300 \mathrm{DPI})$ 

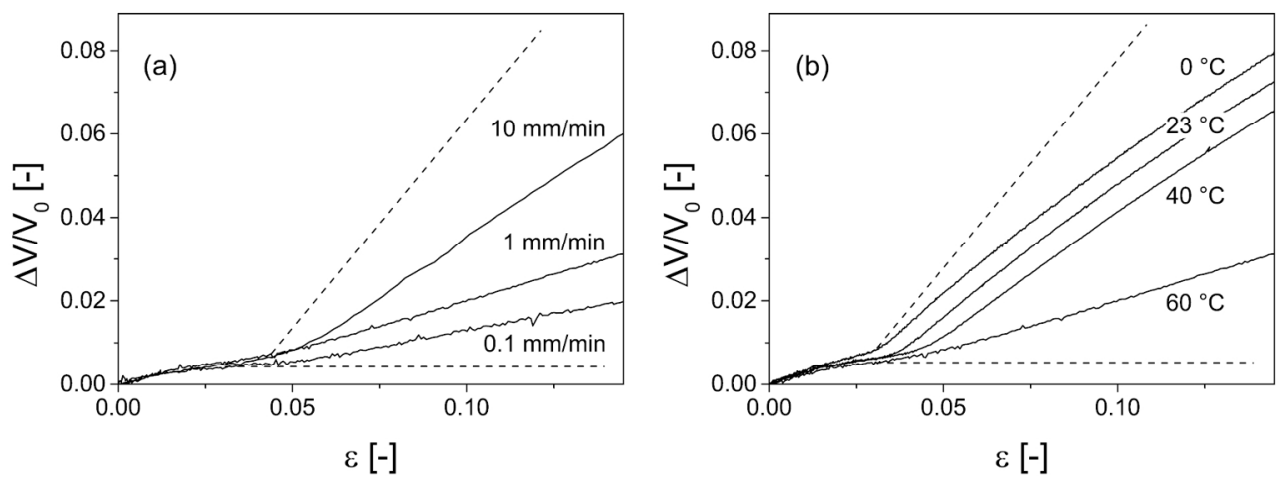

Fig. 10 (a) Volumetric strain vs. longitudinal strain curves at different rates and at fixed temperature of 60 ${ }^{\circ} \mathrm{C}$ for EI resin. (b) Volumetric strain vs. Iongitudinal strain curves at different temperatures and at fixed rate of $1 \mathrm{~mm} / \mathrm{min}$ for EI resin. Dashed lines represent ideal curves with slopes equal to 1 and 0

Fig. 10

$163 \times 60 \mathrm{~mm}(300 \times 300 \mathrm{DPI})$ 